\title{
Digital Health Applications in Heart Failure: a Critical Appraisal of Literature
}

Medhat Farwati, MD

Haris Riaz, MD

W. H. Wilson Tang, $M D^{*}$

\section{Address}

*Department of Cardiovascular Medicine, Heart Vascular and Thoracic Institute, Cleveland Clinic, 9500 Euclid Avenue, Desk J3-4, Cleveland, 0H, 44195, USA

Email: tangw@ccf.org

Published online: 16 January 2021

(C) The Author(s), under exclusive licence to Springer Science+Business Media, LLC part of Springer Nature 2021

This article is part of the Topical Collection on Heart Failure

Keywords Digital health · Remote monitoring

\section{Abstract}

Purpose of the review Despite advancements in the diagnostic and therapeutic armamentarium, heart failure (HF) remains a major public health concern in the USA and worldwide. Digital health applications hold promise to bridge this gap and improve HF care. This review will provide the reader with a concise overview of the current digital health applications in $\mathrm{HF}$, the main challenges to its use, and discuss the future of digital health for promoting care for HF patients.

Recent findings Emerging evidence continues to support the potential role of digital health across the continuum of HF disease process including primary prevention, early detection, disease management, and reducing associated morbidity. There is also increasing emphasis on the need to pursue rigorous investigations to validate these promising claims, with some successful stories that have changed clinical practices.

Summary Digital health technologies have emerged as potentially useful tools to complement HF care in both research and clinical realms. As digital technologies continue to play an increasing role in transforming healthcare delivery, creating the framework for its effective use would be necessary to ensure that digital health applications consistently improve outcomes and enhance care for HF patients. 


\section{Introduction}

Despite advancements in the diagnostic and therapeutic armamentarium, heart failure (HF) remains a major public health concern in the USA and worldwide owing to morbidity, mortality, and economic implications. Given the improved survival of patients and with aging population, the burden of HF has continued to rise [1, 2]. It is estimated that by $2030,>8$ million people $\geq$ 18 years of age will be living with HF, representing $50 \%$ increase in prevalence compared to 2012 [2]. Despite the availability of effective evidence-based treatment options, the prognosis of HF remains poor, with almost half of patients dying within 5 years of initial HF diagnosis [3]. This discrepancy is thought to reflect a persistent gap in the uptake of and adherence to lifesaving therapies for HF patients [4].

Digital health applications hold promise to bridge this gap and improve HF care. Digital health entails the use of various forms of information and communications technology to advance health, while mobile health or "mHealth," a subset of digital health, involves the use mobile wireless technologies to promote health [5]. Emerging evidence continues to support the potential role of digital health across the continuum of HF disease process including primary prevention, early detection, disease management, and reducing associated morbidity [6]. There is also increasing emphasis on the need to pursue rigorous investigations to validate these promising claims, with some successful stories that have changed clinical practices. This review will provide the reader with a concise overview of the current digital health applications in HF with published examples of various categories of diagnostic and intervention strategies. We will discuss the main challenges to its use, and discuss the future of digital health for promoting care for HF patients.

\section{Surveillance of clinical status and stability}

Heart failure exacerbation remains one of the most frequent causes of hospital admissions in the USA with substantial associated costs that continue to rise [7, 8]. Over the years, there is a consensus that various modifiable factors can be ascertained by careful assessment, thus providing opportunity to intervene and prevent such events. A growing field of interest is the potential for digital health to predict decompensation in stable HF patients. Specifically, leveraging digital health applications for monitoring of objective parameters such as weight, voice, hemodynamics, and patient-reported symptoms may improve clinical outcomes in HF patients by allowing early actions to optimize treatment. If proven effective, these applications are expected to help prevent frequent HFrelated hospital admissions and lower healthcare costs. However, the large majority of devices or strategies have largely been designed with the assumption that they may effectively replace current tasks (like manual measurements or inpatient evaluations), and did not subject to rigorous validation.

For the purpose of this review, telemonitoring is broadly defined as the use of information and communication technologies to monitor and transmit information remotely about patients with heart failure.

Numerous studies have investigated the usability of telemonitoring and its impact on outcomes among patients with HF, yielding varying results [9-16]. In a meta-analysis of 14 trials comparing low-profile telemonitoring with usual care in patients with chronic heart failure, remote monitoring programs (the 
majority with telephone communications coupled with consumer devices such as scales and blood pressure cuffs) reduced HF-related admissions by $21 \%$ and all-cause mortality by $20 \%$. In addition, a number of the trials included in that analysis showed reduced costs and improved quality of life measures in the telemonitoring arm [17]. Similarly, in a meta-analysis by Klersy et al., telemonitoring programs were found to be associated with reduced mortality and lower rates of HF-related hospitalizations [18]. In a comprehensive metaanalysis that assessed 15 systematic reviews published between 2003 and 2013, home telemonitoring interventions were associated with reduced risk of allcause mortality ( 0.60 to 0.85 ) and HF-related hospital admissions (0.64 to 0.86) compared with standard of care [19]. In contrast, a large randomized controlled trial of 1653 patients recently hospitalized for heart failure showed that telemonitoring did not improve outcomes including readmission for any reason or all-cause mortality within 6 months after enrollment [20]. In light of conflicting findings, a recent meta-analysis suggested that the positive impact of telemonitoring on outcomes might be dependent on the duration of monitoring [21]. In this study, a reduction of all-cause mortality in the telemonitoring group was observed at 6 months but this effect waned at 1 year. Several factors might contribute to the heterogeneity of the findings related to the impact of telemonitoring on outcomes for HF patients. These include differences in study design and methods, discrepancies in the definition of the control groups, differences in the characteristics of the individual intervention studied, and rapid advances in our technology.

Objective assessments: from implantable to wearable devices

One of the biggest challenges of telemonitoring is the ability to acquire objective data to assess patient status or treatment responses. Heart failure therapy guided by remote monitoring of signs, symptoms, weight, and thoracic impedance biomarkers with cardiac implantable electronic devices (CIED) has not been shown to improve overall clinical outcomes, even when incorporated into remote telemedicine systems [22]. The failure of these technologies may be due to the limitations of the data assessed, rather than the concept of remote monitoring. Nevertheless, recent results from the non-randomized, multicenter MultiSENSE (Multisensor Chronic Evaluation in Ambulatory Heart Failure Patients) study has supported the prognostic value of integrating devicedetected data with heart sounds, respiration, thoracic impedance, heart rate, and activity data. Specifically, the daily index calculated from the HeartLogic algorithm, when combined with standard biomarkers, demonstrated incremental risk stratification for patients with CIED [23]. However, prospective randomized controlled trial on HeartLogic algorithm has not been completed.

The evolution of implantable hemodynamic monitoring has paved the way to achieve that transition [24]. In the CHAMPION (CardioMEMS Heart Sensor Allows Monitoring of Pressure to Improve Outcomes in NYHA Class III Heart Failure Patients) trial [25], using implantable devices for remote monitoring of intracardiac and pulmonary artery (PA) pressures has been shown to significantly reduce HF hospitalizations by $28 \%$ compared to the placebo group. This benefit is thought to be mediated by the ability of clinicians to adjust goaldirected medical therapy in reaction to PA pressures before patients experience symptoms of worsening HF in a more frequent and purposeful manner, leading 
to fewer hospitalizations. Clinical development of various implantable hemodynamic devices measuring left atrial pressure (V-LAP, Vectorious Medical Technologies, Tel Aviv, Israel) or pulmonary artery pressure (Cordella, Endotronix Inc., Lisle, Illinois) are currently ongoing.

Technological advances have paved the way to less invasive technologies that can achieve similar purposes but with broader clinical applicability. The Remote Dielectric Sensing (ReDS) System, which includes a wearable vest and 2 sensors, allows for noninvasive measurement of patient lung fluid content. Low power electromagnetic signals are emitted and intercepted via the sensors reflecting lung fluid volume based on differences in dielectric coefficients between water (high) and air (low). The generated information is automatically saved onto a cloud allowing clinicians to adjust treatment in response to early signals of fluid accumulation. This technique has been leveraged to monitor lung fluid content in HF patients. The ReDS system was first assessed in a prospective single-arm study of 50 patients hospitalized for acute decompensated HF, which demonstrated reduction of hospital readmission with ReDSguided management by $87 \%$ compared to historical controls [26]. The results are largely replicated in the SMILE study that evaluated at-home use among 268 patients with HF with a mean patient follow-up of $6.1 \pm 3.4$ months. Specifically, ReDS-guided HF management was associated with a 58\% reduction in HF readmission [27].

Device analytics have also evolved beyond traditional physiologic measurements for clinical interpretation to applications of artificial intelligence (particularly deep learning technologies) with continuous analytics for risk stratification purposes. The LINK-HF study tested a low-profile disposable adhesive sensor patch with a 7-day disposable battery and a reusable electronic module that is transmitted via Bluetooth to a smartphone that connects with a continuous data collection platform [28]. This device records continuous ECG, continuous 3-axis accelerometry, skin impedance, skin temperature, heart rate, heart rate variability, arrhythmia burden, respiration, activity, sleep, body tilt, and body posture to measure patient-level changes. This patient-monitoring system was able to detect vulnerability signals by a median of 6.5 days prior to hospitalization. Such individualized risk profiling may improve diagnostic accuracy and provide adequate lag time to intervene and execute preventive measures. Nonetheless, the LINK-HF findings should be interpreted in light of the study's significant limitations including a small sample size (100 patients with only 2 females) and lack of external validation.

Another promising strategy has evolved with technological advances with the development of vocal biomarkers with voice signal analysis. In a pilot study of 10 cases, voices of patients were analyzed as they were undergoing inpatient treatment for acute decompensated HF [29]. Using an acoustic microphone and neck-mounted accelerometer, vocal changes were tracked as patients' volume status changed. The authors found that several measures of voice quality may correlate well with HF volume status and have the potential to be used to monitor stable patients at home to predict impending decompensation. Recently, using a large cohort of HF patients who were registered to a call center in Israel, noninvasive voice signal characteristics were independently associated with adverse clinical outcome such as death and HF hospitalizations [30].

Jugular venous pulse assessment with wearable noninvasive devices is also a promising approach, although current devices are yet to be deployed for patient 
home use. Amelard et al. demonstrated the feasibility of using photoplethysmographic imaging system to monitor the jugular venous pressure noninvasively [31]. This technology uses an illumination source and a photodetector at the skin surface to assess the venous blood flow in the jugular vein at the neck level. Clinical application of this technology may provide clinicians with actionable information related to HF status via non-contact ambulatory monitoring of central venous pressure.

Weight monitoring is frequently recommended by healthcare providers for HF patients to predict exacerbation and guide management. Adherence to regular self-weighing is variable with some studies suggesting that less than half of HF patients reported compliance with daily weight measurement [32]. Wearable biosensors that can be integrated into shoes might represent an effortless method for continuous weight monitoring in HF patients and provide the opportunity for weight-based timely intervention while patients are still at home or in the ambulatory setting [33].

While the above-discussed strategies are promising, future research should focus on addressing the limitations of the initial studies testing these new technologies including relatively small sample size and the lack of independent validation. While these technologies continue to evolve, further validation is needed before widespread use in clinical practice.

\section{Interventions to enhance adherence and modify healthy behaviors}

Management of HF involves complex medication regimen as well as ongoing selfdriven dedication to behavioral modification and self-care. However, patients' failure to adhere to evidence-based pharmacologic interventions has been well described [34, 35]. Medication non-adherence in HF patients is a multifactorial issue and various strategies have been implemented to address this problem with variable success. As technology continues to play an ever-increasing role in patients' daily routine, digital health creates the opportunity to improve adherence to treatment in HF patients leading to better outcomes. The impact of digital technologies on medication adherence and physical activity has been investigated in several chronic diseases, yielding conflicting results [36-38]. In a meta-analysis by Thakkar et al., mobile phone text messages were found to improve self-reported medication adherence in the context of various chronic diseases including cardiovascular disease [38]. Similarly, the tobacco, exercise, and diet messages (TEXT ME) trial demonstrated a modest improvement in cardiovascular risk factors (e.g., lipid control, blood pressure, and body mass index) with the use of a lifestyle-focused text messaging service in patients with coronary heart disease. In a single-center demonstration project that enrolled $60 \mathrm{HF}$ patients with remote monitoring of blood pressure and weight with two interactive texting-based mobile apps in the 30-day high-risk period post-discharge through a cloud-based HIPAA compliant platform, a promising 50\% 30-day readmission rate reduction was achieved, with $73 \%$ active patient engagement beyond the initial 30-day period [13]. These studies demonstrate that leveraging widely utilized mobile technologies with effective feedback and engagement incentives are potentially successful strategies to consider. 
Virtual visits have been popularized upon necessity with the recent challenges related to patient care management during the COVID-19 pandemic. Nevertheless, broad technological adoption of teleconference capabilities has enabled such virtual patient encounters to be effective patient management tools [39]. Recently, a single-center, non-blinded, prospective randomized pilot study evaluating the role of virtual visit following discharge from HF demonstrated safety (similar 45-day clinical event rates between groups) but nonsignificant lower no-show rates with 7-day virtual visits when directly compared to in-person visits (albeit with favorable numerical trends towards virtual visit arms) [40]. An important observation is that technological limitations were observed only in $10 \%$ of patients included in the study, illustrating the potential broad applicability of such strategy.

On the contrary, not all behavioral management programs achieve effective results especially when new devices outside current self-management practices are required. The Innovative Approaches to Diet, Exercise, and Activity (IDEA) trial showed that wearable technology devices combined with standard behavioral weight loss approach resulted in less weight loss over 2 years compared with what was achieved with the standard intervention alone [37]. It is important to note, however, that patients with HF were not enrolled in the abovementioned trials highlighting the scarcity of HF-specific studies. In a pilot randomized controlled trial of 29 patients, Hale et al. investigated the use of an innovative telehealth-based technology to improve medication adherence for HF patients [41]. The intervention is a remote medication monitoring system that comprised a home-based device that alerts patients about the time of their daily medications and a monitoring center with personnel who contact patients and providers when medications are not taken. Notably, no significant difference in self-reported medication adherence was observed between the two arms. However, a reduction of $80 \%$ in the risk of all-cause hospitalization and a significant decrease in the number of all-cause hospitalizations were observed in the intervention group.

\section{Novel clinical endpoints}

Digital technology offers the opportunity to collect novel endpoints and is being increasingly recognized as a potentially useful component of clinical trials. Moreover, digital technology can be part of the administered intervention and the collected endpoint. The TARGET-HF-DM (Technologies to improve drug Adherence and Reinforce Guideline based Exercise Targets in patients with Heart Failure and Diabetes Mellitus) trial is currently evaluating whether a mHealth intervention might improve health behaviors and outcomes in this high-risk population [42]. Namely, this trial is set out to determine the efficacy of a wearable step counter (Withings Go, Issy-les-Moulineaux, France), combined with personalized feedback via text messages and a medication adherence teaching tool, in improving physical activity and drug adherence for patients with concomitant HF and diabetes mellitus. Disease status will be quantified by physiologic parameters such as NT-proBNP and HbA1c. Interestingly, TARGETHF DM trial [42], the intervention comprised a wearable step counter combined with personalized text messages based on step count data. The same step counter was leveraged to ascertain the primary endpoint for physical activity 
that is change in weekly step count. In the landmark NEAT-HFpEF (Nitrate's Effect on Activity Tolerance in Heart Failure With Preserved Ejection Fraction), digital technology was used to generate novel endpoints [43]. In that trial, 110 patients with heart failure with preserved ejection fraction were randomized to a 6-week regimen of isosorbide mononitrate or placebo with subsequent crossover to the other group. The primary endpoint, changes in daily activity, was measured via patient-worn belt outfitted accelerometers. This technology was selected as it offers the opportunity for continuous assessment of daily physical activity compared with intermittent evaluation of exercise tolerance obtained by traditional measures such as the 6-min walk test. By providing continuous dense data that complement traditional markers, these novel biomarkers might offer additional insights on the effects of interventions tested in clinical trials.

\section{Challenges and future directions}

Successful incorporation of digital health technologies in HF care is faced by several barriers that may vary across different stakeholders including patients and providers as well as challenges inherent to the structure of healthcare system. It is important to note that most of these challenges are related to the uptake of digital health technologies in general and not necessarily specific to HF care. Figure 1 outlines various opportunities and barriers/challenges in pateint-centered HF care in the digital health ecosystem.

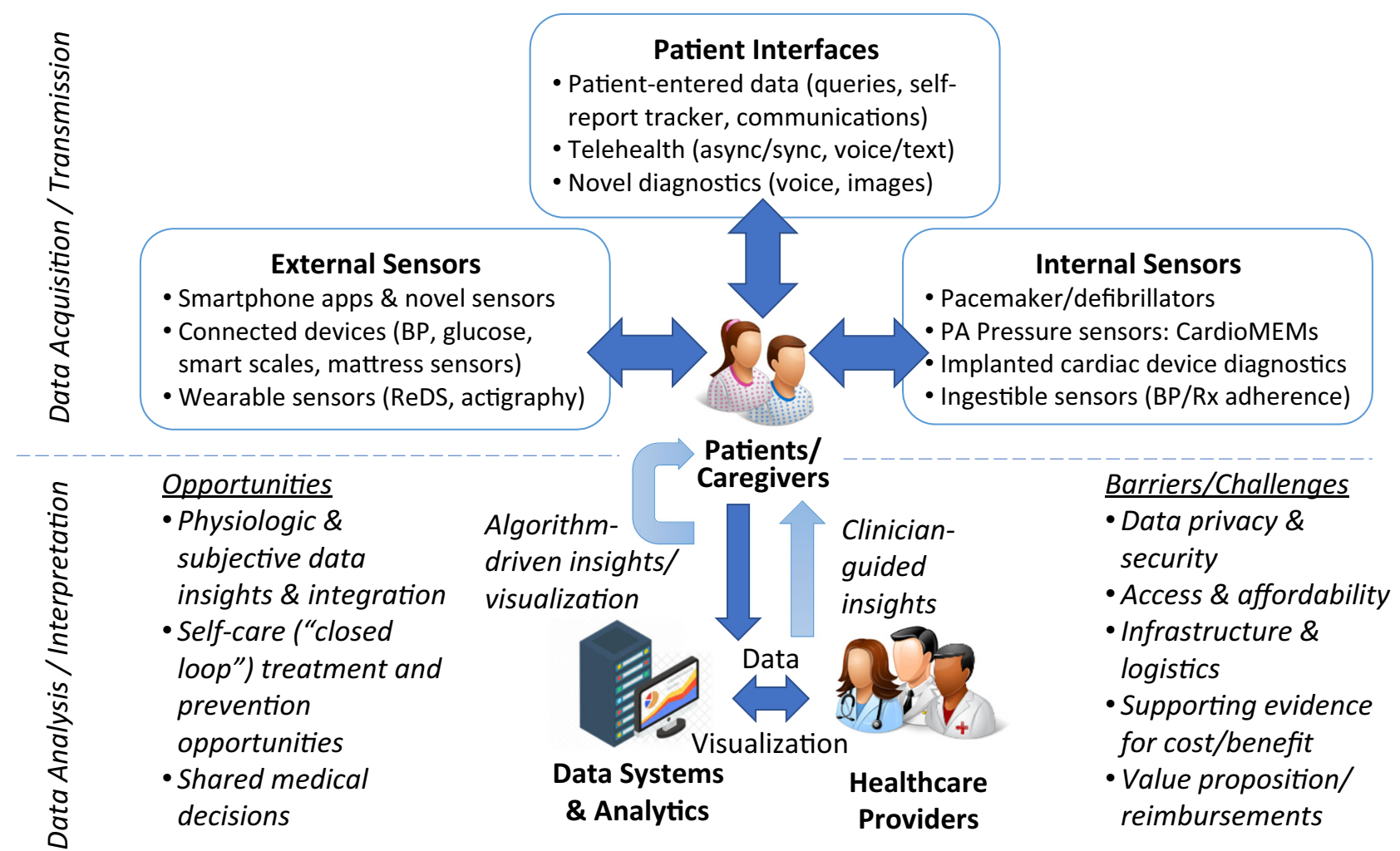

Figure 1. The Heart Failure Digital Health Ecosystem: Patient-Centric Care Components, Opportunities, and Barriers/Challenges. 
From a patient standpoint, data safety and privacy is a valid concern. Multiple studies have suggested that many patients continue to worry about the security of their medical information and may have little confidence in the ability of the current technology to safeguard their privacy [44-46]. In a US survey administered to 3959 adults between October 2011 and February 2012, about two-thirds of respondents expressed their concern about the safety of their protected health information [45]. Moreover, public trust in large data security is likely to be negatively affected by the recent waves of data breaches from commercial corporations such as Facebook and Amazon as well as medical device companies including defibrillators and pacemakers data [47]. These concerns need to be thoughtfully acknowledged and addressed in the development of digital health technologies. While relatively new technologies such as smartphones and tablets are becoming increasingly ubiquitous, a sizable minority of HF patients (elderly, in particular) do not own smart phones and might lack access to these novel digital health interventions [48]. In addition, not all patients will be able to afford certain commercial wearables (e.g., Apple Watch) creating the potential for aggravating existing disparities in HF care. Professional societies, healthcare policy makers, and patient advocacy groups need to join forces to ensure equitable access to these technologies across various socioeconomic and racial groups. Adequate health or technological literacy and self-care capabilities may also hamper digital health implementation at the patient level, especially in those with social challenges in healthcare. Each patient has his or her unique needs with clinically accurate and actionable feedbacks, highlighting the importance of tailoring digital health strategies to individual needs.

For healthcare providers, caring for HF patients is already a complex task that requires processing of large amount data in order to optimize medical management for these patients. Consolidation of health information is burdensome especially for non-traditional clinical parameters or those that have been acquired with lower confidence. Many providers are concerned that simply adding large volumes of data from digital health applications (e.g., sensors and devices) might become overwhelming and paradoxically lead to inefficient and poorer care. As such, linking these extensive datasets to actionable tasks would be of paramount importance to streamline the physicians' care delivery and support, rather than hinder, the clinical decision-making at the point of care. Communications and clinical implementation have to be efficient and aligned to clinical workflow. Early partnership and mutual incentives between clinicians and developers of digital health technologies would be key to establish the optimal methods for incorporating data generated from these new technologies into the providers' workflow. This vision has been also endorsed by the most recent American College of Cardiology statement on healthcare transformation in the era of digital health and big data [49].

The current healthcare system has also posed unique challenges on the uptake and utilization of digital health applications for HF patients. Frequently, the existing healthcare infrastructure does not support the integration or expense of novel digital health technologies. Specifically, most of the current electronic health records (EHRs) are not equipped to pull data directly from novel technologies limiting the optimal use of the 


\section{Conclusions}

generated data. Enhancing the interoperability between the commonly used EHRs and the rapidly expanding new devices and wearables should be prioritized and would require close collaboration between technology developers and EHRs vendors.

From a payer's perspective, guidelines for reimbursement related to the use of digital health applications in practice are not well established. In part, this is due to the need for rigorous efficacy and cost-effectiveness data as well as lack of clear standards of evidence required for reimbursement of these novel technologies. Thus, high-quality evidence establishing the value and efficacy of novel technologies would be needed before such technologies be eligible for coverage under the current reimbursement models. Even when there is solid evidence supported by randomized controlled trials and clinical evidence (such as CardioMEMs device), payers may still deny coverage of costly interventions due to business or programmatic priorities in an environment limited overall healthcare resources.

Digital health technologies have emerged as potentially useful tools to complement HF care across the continuum of HF disease process including primary prevention, early detection, disease management, and reducing associated morbidity. In this review, we presented the current digital health applications in HF in both research and clinical realms, discussed the main challenges to its use from various stakeholders' perspective, and shed some light on the future of digital health for advancing care for HF patients. As digital technologies continue to play an increasing role in transforming healthcare delivery, creating the framework for its effective use would be necessary to ensure that digital health applications consistently improve outcomes and enhance care for HF patients.

\section{Compliance with Ethical Standards}

\section{Conflict of interest}

Medhat Farwati declares that he has no conflict of interest.

Haris Riaz declares that he has no conflict of interest.

W. H. Wilson Tang declares that he has no conflict of interest. Dr. Tang is partially supported by research grant from the National Institutes of Health (R01HL126827).

Human and animal rights and informed consent

This article does not contain any studies with human or animal subjects performed by any of the authors.

\section{References Recommeding Reading}

1. Barker WH, Mullooly JP, Getchell W. Changing incidence and survival for heart failure in a well-defined older population, 1970-1974 and 1990-1994. Circulation. 2006;113(6):799-805.
2. Virani SS, Alonso A, Benjamin EJ, Bittencourt MS, Callaway CW, Carson AP, et al. Heart disease and stroke statistics - 2020 update: A reportfrom the 
American Heart Association. Circulation 2020;141(9): e139-e596.

3. Roger VL, Weston SA, Redfield MM, HellermannHoman JP, Killian J, Yawn BP, et al. Trends in heart failure incidence and survival in a community-based population. Jama. 2004;292(3):344-50.

4. Greene SJ, Butler J, Albert NM, DeVore AD, Sharma PP, Duffy CI, et al. Medical therapy for heart failure with reduced ejection fraction: the CHAMP-HF registry. J Am Coll Cardiol. 2018;72(4):351-66.

5. World Health Organization. WHO guideline: recommendation on digital interventions for health system strengthening: web supplement 2: summary offindings and GRADE tables. World Health Organization; 2019. Accessed on January 10, 2021 athttps://www.who.int/ reproductivehealth/publications/digital-interventionshealth-system-strengthening/en/

6. DeVore AD, Wosik J, Hernandez AF. The future of wearables in heart failure patients. JACC Hear Fail. 2019;7(11):922-32.

7. Krishnamurthi N, Francis J, Fihn SD, Meyer CS, Whooley MA. Leading causes of cardiovascular hospitalization in 8.45 million US veterans. PLoS One. 2018;13(3):e0193996.

8. Heidenreich PA, Albert NM, Allen LA, Bluemke DA, Butler J, Fonarow GC, et al. Forecasting the impact of heart failure in the United States: a policy statement from the American Heart Association. Circ Heart Fail. 2013;6(3):606-19.

9. Zai AH, Ronquillo JG, Nieves R, Chueh HC, Kvedar JC, Jethwani K. Assessing hospital readmission risk factors in heart failure patients enrolled in a telemonitoring program. Int J Telemed Appl. 2013;2013:305819.

10. Rosen D, McCall JD, Primack BA. Telehealth protocol to prevent readmission among high-risk patients with congestive heart failure. Am J Med.

2017;130(11):1326-30.

11. Chaudhry SI, Phillips CO, Stewart SS, Riegel B, Mattera JA, Jerant AF, et al. Telemonitoring for patients with chronic heart failure: a systematic review. J Card Fail. 2007;13(1):56-62.

12. Bashi N, Karunanithi M, Fatehi F, Ding H, Walters D. Remote monitoring of patients with heart failure: an overview of systematic reviews. J Med Internet Res. 2017;19(1):e18.

13. Park C, Otobo E, Ullman J, Rogers J, Fasihuddin F, Garg S, et al. Impact on readmission reduction among heart failure patients using digital health monitoring: feasibility and adoptability study. JMIR Med Inform. 2019;7(4):e13353.

14. Maric B, Kaan A, Ignaszewski A, Lear SA. A systematic review of telemonitoring technologies in heart failure. Eur J Heart Fail. 2009;11(5):506-17.

15. Zan S, Agboola S, Moore SA, Parks KA, Kvedar JC, Jethwani K. Patient engagement with a mobile webbased telemonitoring system for heart failure selfmanagement: a pilot study. JMIR mHealth uHealth. 2015;3(2):e33.
16. Evans J, Papadopoulos A, Silvers CT, Charness N, Boot WR, Schlachta-Fairchild L, et al. Remote health monitoring for older adults and those with heart failure: adherence and system usability. Telemed e-Health. 2016;22(6):480-8.

17. Clark RA, Inglis SC, McAlister FA, Cleland JGF, Stewart $\mathrm{S}$. Telemonitoring or structured telephone support programmes for patients with chronic heart failure: systematic review and meta-analysis. Bmj. 2007;334(7600):942.

18. Klersy C, De Silvestri A, Gabutti G, Regoli F, Auricchio A. A meta-analysis of remote monitoring of heart failure patients. J Am Coll Cardiol. 2009;54(18):1683-94.

19. Kitsiou S, Pare G, Jaana M. Effects of home telemonitoring interventions on patients with chronic heart failure: an overview of systematic reviews. J Med Internet Res. 2015;17(3):e63.

20. Chaudhry SI, Mattera JA, Curtis JP, Spertus JA, Herrin J, Lin $Z$, et al. Telemonitoring in patients with heart failure. N Engl J Med. 2010;363(24):2301-9.

21. Pekmezaris R, Tortez L, Williams M, Patel V, Makaryus A, Zeltser R, et al. Home telemonitoring in heart failure: a systematic review and meta-analysis. Health Aff. 2018;37(12):1983-9.

22. Samara MA, Tang WHW. Device monitoring strategies in acute heart failure syndromes. Heart Fail Rev. 2011;16(5):491-502.

23. Gardner RS, Singh JP, Stancak B, Nair DG, Cao M, Schulze C, et al. HeartLogic multisensor algorithm identifies patients during periods of significantly increased risk of heart failure events: results from the MultiSENSE study. Circ Heart Fail. 2018;11(7):e004669.

24. Abraham WT, Perl L. Implantable hemodynamic monitoring for heart failure patients. J Am Coll Cardiol. 2017;70(3):389-98.

25. Givertz MM, Stevenson LW, Costanzo MR, Bourge RC, Bauman JG, Ginn G, et al. Pulmonary artery pressureguided management of patients with heart failure and reduced ejection fraction. J Am Coll Cardiol. 2017;70(15):1875-86.

26. Amir O, Ben-Gal T, Weinstein JM, Schliamser J, Burkhoff D, Abbo A, et al. Evaluation of remote dielectric sensing (ReDS) technology-guided therapy for decreasing heart failure re-hospitalizations. Int J Cardiol. 2017;240:279-84.

27. Abraham WT, Anker S, Burkhoff D, Cleland J, Gorodeski E, Jaarsma T, et al. Primary results of the Sensible Medical Innovations Lung Fluid Status Monitor allows reducing readmission rate of heart failure patients (SMILE) trial. J Card Fail. 2019;25(11):938.

28. Stehlik J, Schmalfuss C, Bozkurt B, Nativi-Nicolau J, Wohlfahrt P, Wegerich S, et al. Continuous wearable monitoring analytics predict heart failure hospitalization: the LINK-HF multicenter study. Circ Heart Fail. 2020;13(3):e006513.

29. Murton OM, Hillman RE, Mehta DD, Semigran M, Daher M, Cunningham T, et al. Acoustic speech 
analysis of patients with decompensated heart failure: a pilot study. J Acoust Soc Am. 2017;142(4):EL401-7.

30. Maor E, Perry D, Mevorach D, Taiblum N, Luz Y, Mazin I, et al. Vocal biomarker is associated with hospitalization and mortality among heart failure patients. J Am Heart Assoc. 2020;9(7):e013359.

31. Amelard R, Hughson RL, Greaves DK, Pfisterer KJ, Leung J, Clausi DA, et al. Non-contact hemodynamic imaging reveals the jugular venous pulse waveform. Sci Rep. 2017;7:40150.

32. Lainščak M, Cleland JGF, Lenzen MJ, Nabb S, Keber I, Follath F, et al. Recall of lifestyle advice in patients recently hospitalised with heart failure: a EuroHeart Failure Survey analysis. Eur J Heart Fail. 2007;9(11):1095-103.

33. Elian A, Sada N, Elfadel I, Saeed M, Shabra A. A preliminary evaluation of continuous, shoe-integrated weight measurements for heart failure patients. In: 2016 38th Annual International Conference of the IEEE Engineering in Medicine and Biology Society (EMBC). IEEE; 2016. p. 4768-71.

34. Wolf MS, King J, Wilson EAH, Curtis LM, Bailey SC, Duhig J, et al. Usability of FDA-approved medication guides. J Gen Intern Med. 2012;27(12):1714-20.

35. Granger BB, Ekman I, Hernandez AF, Sawyer T, Bowers MT, DeWald TA, et al. Results of the Chronic Heart Failure Intervention to Improve MEdication Adherence study: a randomized intervention in high-risk patients. Am Heart J. 2015;169(4):539-48.

36. Chow CK, Redfern J, Hillis GS, Thakkar J, Santo K, Hackett ML, et al. Effect of lifestyle-focused text messaging on risk factor modification in patients with coronary heart disease: a randomized clinical trial. Jama. 2015;314(12):1255-63.

37. Jakicic JM, Davis KK, Rogers RJ, King WC, Marcus MD, Helsel D, et al. Effect of wearable technology combined with a lifestyle intervention on long-term weight loss: the IDEA randomized clinical trial. Jama. 2016;316(11):1161-71.

38. Thakkar J, Kurup R, Laba T-L, Santo K, Thiagalingam A, Rodgers A, et al. Mobile telephone text messaging for medication adherence in chronic disease: a meta-analysis. JAMA Intern Med. 2016;176(3):340-9.

Available from: https://doi.org/10.1001/ jamainternmed.2015.7667.

39. Gorodeski EZ, Goyal P, Cox ZL, Thibodeau JT, Reay RE, Rasmusson K, et al. Virtual visits for care of patients with heart failure in the era of COVID-19: a statement from the Heart Failure Society of America. J Card Fail. 2020;26(6): 448-56.

40. Gorodeski EZ, Moennich LA, Riaz H, Jehi L, Young JB, Tang WHW. Virtual versus in-person visits and appointment no-show rates in heart failure care transitions. Circ Hear Fail. 2020; published online on July 27, 2020. https://doi.org/10.1161/ CIRCHEARTFAILURE.120.007119.
41. Hale TM, Jethwani K, Kandola MS, Saldana F, Kvedar JC. A remote medication monitoring system for chronic heart failure patients to reduce readmissions: a two-arm randomized pilot study. J Med Internet Res. 2016;18(5):e91

42. Sharma A, Mentz RJ, Granger BB, Heitner JF, Cooper LB, Banerjee D, et al. Utilizing mobile technologies to improve physical activity and medication adherence in patients with heart failure and diabetes mellitus: rationale and design of the TARGET-HF-DM trial. Am Heart J. 2019;211:22-33.

43. Redfield MM, Anstrom KJ, Levine JA, Koepp GA, Borlaug BA, Chen $\mathrm{HH}$, et al. Isosorbide mononitrate in heart failure with preserved ejection fraction. N Engl J Med. 2015;373(24):2314-24.

Available from: https://doi.org/10.1056/

NEJMoa1510774.

44. Papoutsi C, Reed JE, Marston C, Lewis R, Majeed A, Bell D. Patient and public views about the security and privacy of Electronic Health Records (EHRs) in the UK: results from a mixed methods study. BMC Med Inform Decis Mak. 2015;15(1):86.

45. Agaku IT, Adisa AO, Ayo-Yusuf OA, Connolly GN. Concern about security and privacy, and perceived control over collection and use of health information are related to withholding of health information from healthcare providers. J Am Med Inform Assoc. 2013;21(2):374-8.

Available from: https://doi.org/10.1136/amiajnl2013-002079.

46. Giokas D. Canada health infoway-towards a national interoperable electronic health record (EHR) solution. Stud Health Technol Inform. 2005;115:108-40.

47. Newman LH. Medical devices are the next security nightmare. WIRED. 2017; Accessed on 1/10/2021 at https://www.wired.com/2017/03/medicaldevicesnext-security-nightmare.

48. Mobile fact sheet. More fact sheets: Internet/ Broadband J Social Medial. Accessed on 1/10/2021 at https://www.pewresearch.org/internet/factsheet/ mobile/.

49. Bhavnani SP, Parakh K, Atreja A, Druz R, Graham GN, Hayek SS, et al. 2017 Roadmap for innovation-ACC health policy statement on healthcare transformation in the era of digital health, big data, and precision health: a report of the american college of cardiology task force on health policy statements and systems of care. J Am Coll Cardiol. 2017;70(21):2696-718.

\section{Publisher's Note}

Springer Nature remains neutral with regard to jurisdictional claims in published maps and institutional affiliations. 